\title{
Preparation of poly(thiourethane) thermosets by controlled thiol-isocyanate click reaction using a latent organocatalyst
}

\author{
Francesco Gamardella1, Xavier Ramis², Silvia De la Flor ${ }^{3}$, Àngels Serra1 ${ }^{\star *}$ \\ ${ }^{1}$ Dept.of Analytical and Organic Chemistry, Universitat Rovira i Virgili, C/ Marcel.lí Domingo, 43007, Tarragona, Spain \\ 2 Thermodynamics Laboratory, ETSEIB Universitat Politècnica de Catalunya, Av. Diagonal, 08028, Barcelona, Spain \\ ${ }^{3}$ Department of Mechanical Engineering, Universitat Rovira i Virgili, Av. Països Catalans, 26, 43007 Tarragona, Spain \\ *Corresponding author: e-mail: angels.serra@urv.cat
}

\begin{abstract}
s
Different poly(thiourethane) thermosets were prepared by means of a thiol-isocyanate click reaction starting from three diisocyanates with different structure (isophorone diisocyanate, IPDI, 4,4'-methylene bis(cyclohexy isocyanate), HMDI and hexamethylene diisocyanate, HDI, and a tetrathiol, pentaerythritol tetrakis(3-mercaptopropionate), PETMP. The curing process has been catalyzed by basic and acid catalysts. The use of a thermally activated base generator (1methylimidazolium tetraphenylborate, BG1MI), which is an organocatalyst, allowed a better control on the curing initiation than those traditionally used dibutyltin dilaurate (DBTDL) or tertiary amines. The curing evolution was investigated by DSC and FTIR spectroscopy.

The materials obtained were characterized by thermomechanical and mechanical tests. Homogeneous materials were obtained in all cases. The chemical structure of the network was correlated with the thermal and mechanical data determined. $\mathrm{T}_{\mathrm{g}}$ values in the range of 75 to $150^{\circ} \mathrm{C}$ were obtained. The thermal degradation of these materials has also been investigated and a complex degradation mechanism, with three different steps was observed in all cases. These materials showed a higher thermal stability than their oxygen counterparts did.

The thermosets obtained using the latent organocatalyst have similar characteristics to the ones obtained by using the common DBTDL.

\section{Keywords}

Poly(thiourethanes), click reaction, thermosets, latency, organocatalysts.
\end{abstract}




\section{Introduction}

Click-chemistry, introduced for the first time by Kolb et al. in 2001 [1], has attracted a broad interest in the field of thermosets due to its efficiency, selectivity, speed and effective reaction mechanism that leads to the formation of highly homogeneous materials with high crosslinking density and without the formation of unexpected structures and volatile by-products [2]. There are several efficient reactions with different mechanisms that can be considered as click reactions, among them thermal epoxy-amine and Michael addition or photochemical thiol-ene. In addition to those, the nucleophilic addition of thiols to isocyanate satisfied all the criteria required to be considered as "click reaction" [3-6].

Although the reaction that leads to the formation of poly(thiourethanes) (PTU) is known for a long time [7], it has been less investigated than the process leading to poly(urethanes) (PU), their oxygen analogs, which have a great industrial significance reflected in a nearly $5 \%$ of total polymer production [8]. The formation of poly(thiourethanes) is not accompanied by side-reactions often observed in the poly(urethanes) synthesis such as the formation of urethanes and allophanates [9].

Poly(thiourethanes), also named poly(thiocarbamates), are very versatile polymeric materials due to their biocompatibility, excellent physical, mechanical and optical properties. In comparison to poly(urethanes), the incorporation of sulfur to the backbone of the polymeric structure increases flexibility, crystallinity and feasibility to incorporate these structures to polymerizable systems [10,11]. Moreover, the enhanced refractive index makes PTU more suitable for optical applications. Lu et al. prepared PbS/PTU and ZnS/PTU nanocomposites as optical materials with high refractive, index in the range from 1.5 to 2 [12,13]. Moreover, Jaffrennou et al. studied a polythiourethane thermosetting system based on trithiol/diisocyanate formulations for optical applications [14], and Ireni et al. synthesized a poly(thiourethane-urethane-urea) with impressive optical properties, transmittance (>90\%) and a refractive index value of 1.5 [15]. Poly(thiourethane)s have also applied in microfluidics and microparticles preparation [16,17]. Some papers on coatings applications of these materials have also been published [18,19].

In the preparation of poly(urethanes) and poly(thiourethanes), the reaction can be catalyzed by several bases and by a wide variety of Lewis acids [20]. Depending of the characteristics of the catalyst used, the activation occurs through two different ways. When basic catalysts are selected, they activate the alcohol or thiol by forming the corresponding anion (nucleophilic activation) which are the species that attack the isocyanate carbon (see Scheme 1). Alternatively, when Lewis acids are used, they coordinate with the oxygen or nitrogen lone pairs of the isocyanate (electrophilic 
activation) which can be attacked by less nucleophilic agents like neutral thiols or alcohols [21,22]. Generally speaking, thiols are more acidic than alcohols, and the basic catalyst helps to form thiolate anions, with an accentuated nucleophilic character, higher than alkoxides. According to that, the reactivity of thiols is higher than the reactivity of alcohols and subsequently, the attack to the isocyanate is much faster.
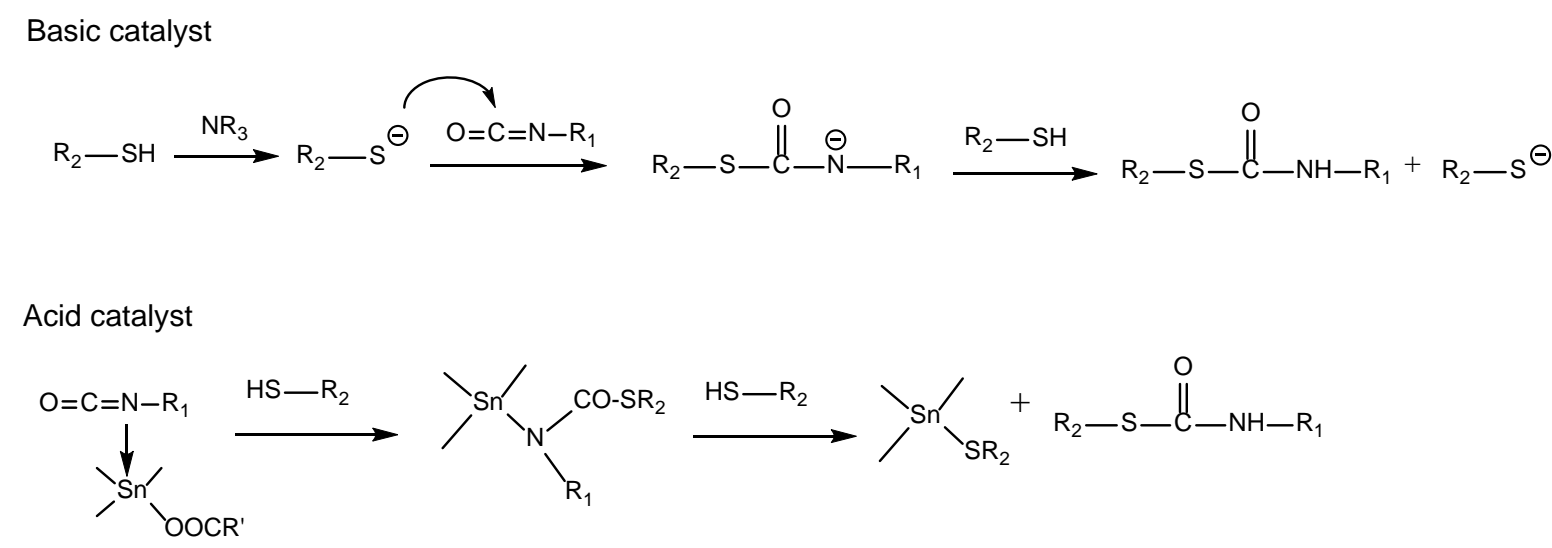

Scheme 1. Proposed mechanisms of poly(thiouretane) formation in both basic and acidic conditions

The aim of the present work is the preparation and characterization of a series of poly(thiouretane) networks and therefore, the curing schedule and conditions must be deeply investigated to reach the most adequate conditions leading to thermosets with high performance. According to that, the selection of the catalyst and its proportion is the key point to reach a high technical applicability in the desired processing time.

The most common basic catalysts involved in the thiol-isocyanate polycondensation are tertiary amines such as triethylamine (TEA) or amidine compounds such as 1,5-diazabicyclo[4.3.0]non-5ene (DBN) or 1,8-diazabicyclo[5.4.0]undec-7-ene (DBU) [23,24]. Recently, Shin et al. reported the use of amines to perform thiol-isocyanate-ene ternary networks by sequential and simultaneous click reactions [25]. The same research team described the synthesis of elastic segmented poly(thiourethane)s by sequential thiol-ene and thiol-isocyanate reactions in the presence of TEA [26]. However, in the presence of basic catalyst, once initiated the reaction is too fast and the system is difficult to process due to the high reactivity of the monomers involved in the reaction. The fast reaction can be attributed to the low pKa of thiol species and their easy deprotonation in presence of the base. Thus, the formation of poly(thiourethane) networks is much faster, and consequently more problematic in terms of processing, than the formation of crosslinked poly(urethanes) $[12,13,27,28]$.

To reduce the speed of the reaction, one of the possibilities is to decrease the amount of catalyst. However, in many studies a low degree of conversion of the monomers involved was obtained, which 
in crosslinked polymers lead to a reduction of the $T_{\mathrm{g}}$ values. The possibilities of using a weaker base or much better, a latent basic catalyst, that release the base after the application of an external stimulus, could be a solution to prepare, in a controlled way, thermosets by thiol-isocyanate clickreactions.

Besides the use of bases, the most common acid catalysts are organometallic tin compounds, especially dibutyltin dilaurate (DBTDL), which has been used by several authors to prepare PTUs for optical applications $[12,13,14]$. Kultys et al. selected DBTDL to synthetize a new copolymeric thermoplastic, poly(thiourethane-urethane)s [29]. It has been described that DBTDL leads to low reaction rates, resulting in long curing times or high curing temperatures, which can negatively affect the performance of the crosslinked materials. In addition to that, we should consider that the European Union have restricted the use of organotin-based compounds. Therefore, the use of organocatalyst in the preparation of polymeric materials is one of the improvements to be fulfilled to accomplish the EU regulations and to open new possibilities of application in the biomedical field [30,31].

In previous studies of our group, the use of latent bases has been explored to catalyzed wellcontrolled crosslinking procedures. LC-80, which is a commercially available encapsulated imidazole has been used in the preparation of thermosets by thiol-epoxy reaction [32]. We have also proved that photolatent bases as tertiaryammonium tetraphenylborates (BG) liberates amines on reaching a certain temperature [33]. Shin et al. described the use of a photolatent base in the preparation of poly(thiourethane)s triggered by UV-irradiation. However, this system has some limitations such as lower curing degree in shadowed areas, possible vitrification of the material and that the photochemical processes are restricted to the preparation of thin layers [34].

In the present study, we have tested latent systems (LC-80 and BG1MI) and we compare their efficiency with DBTDL by means of calorimetric studies. Three different isocyanates, isophorone diisocyanate (IPDI), 4,4'-methylene bis(cyclohexyl isocyanate) (HMDI) and hexamethylene diisocyanate(HDI) and pentaerythritol tetrakis(3-mercaptopropionate) (PETMP) (see Scheme 2) have been used as starting monomers.<smiles>CC1(C)CC([N+](=O)[O-])CC(C)(CN=O)C1</smiles>

IPDI<smiles>O=C=NC1CCC(CC2CCC(N=C=O)CC2)CC1</smiles>

HMDI<smiles>O=C=NCCCCCCCN=C=O</smiles>

HDI

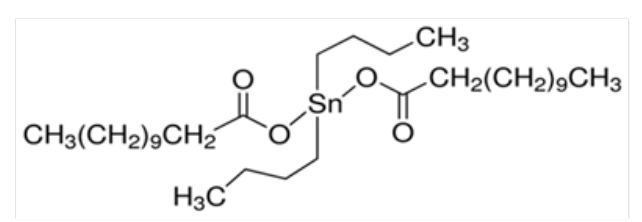

DBTDL

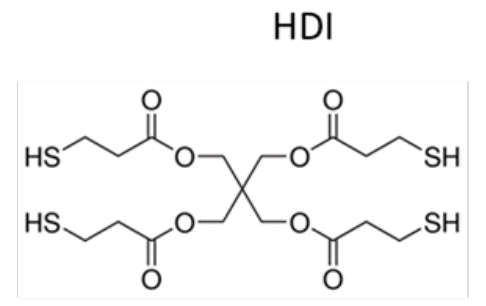

PETMP 
<smiles>c1ccc([B-](c2ccccc2)(c2ccccc2)c2ccccc2)cc1</smiles>

BG1MI<smiles>c1c[nH]cn1</smiles>

Scheme 2. Structure of the starting compounds

The mechanical and thermal properties of the resultant poly(thiourethane) thermosets have been investigated by thermomechanical and thermogravimetric analysis. Mechanical tests have also been performed.

\section{Experimental Methods}

\subsection{Materials}

Pentaerythritol tetrakis(3-mercaptopropionate) (PETMP), dibutyltin dilaurate (DBTDL) from Sigma-Aldrich. Isophorone diisocyanate (IPDI) and 4,4'-methylene bis(cyclohexyl isocyanate) (HMDI) from Acroos Organics and hexamethylene diisocyanate (HDI) from Sigma-Aldrich. Amine precursors were Technicure ${ }^{\circledR}$ LC-80 (encapsulated imidazole) from AC Catalysts. We synthesized the base generator, 1-methylimidazolium tetraphenylborate (BG1MI), according to a reported methodology [,35] and its characterization was performed by FTIR and ${ }^{1} \mathrm{H}-\mathrm{NMR}$ spectroscopy (see supporting information).

\subsection{Preparation of the formulations}

The different formulations were prepared by adding the corresponding amount of diisocyanate, to a solution of the selected amount of LC80 or BG1MI in the thiol. Isocyanate and thiol groups are mixed in stoichiometric proportions. In case of using DBTDL as the catalyst, it was first dissolved in the isocyanate and the corresponding amount of thiol was added. The different catalysts were added in several proportions 0.5, 1 and 2 phr, in reference to the thiol amount (parts of catalysts per hundred parts of thiol).

The thiol-catalyst mixtures were manually stirred until homogeneity at room temperature, with the exception of BG1MI, in which a temperature of $80^{\circ} \mathrm{C}$ and magnetic stirring was required. This mixture was cooled down and then the diisocyanate was added. The samples were studied freshly prepared. 


\subsection{Sample preparation}

For DMTA analysis, the prepared formulations were poured onto aluminium moulds and cured sequentially at $80,100,130$ and $165^{\circ} \mathrm{C}$ one hour at each temperature.

For tensile test, films were prepared by pouring the formulations between two glasses (protected with an adhesive sheet of Teflon) and using Teflon spacers to ensure a homogeneous thickness of 0.4 $\mathrm{mm}$. The curing schedule applied was as before and a post-curing of $3 \mathrm{~h}$ at $170^{\circ} \mathrm{C}$ was done to reach the complete curing. The films were die-cut to obtain Type V specimens adapted for ASTM D63814 requirements.

\subsection{Characterization techniques}

A differential scanning calorimeter (DSC) Mettler DSC-3+ calibrated using an indium standard (heat flow calibration) and an indium-lead-zinc standard (temperature calibration) was used to analyze the curing evolution. Samples of approximately 5-10 mg were tested in aluminum pans with a pierced lid in a nitrogen atmosphere with a gas flow of $50 \mathrm{~mL} / \mathrm{min}$. The dynamic studies were performed in a temperature range of $30-250{ }^{\circ} \mathrm{C}$ with a heating rate of $10^{\circ} \mathrm{C} / \mathrm{min}$. The enthalpy $(\Delta H)$ released during curing of the samples was calculated by integration of the calorimetric signal using a straight baseline, with the help of the STARe software.

${ }^{1} \mathrm{H}$ NMR spectra were registered in a Varian Gemini 400 spectrometer. $\mathrm{CDCl}_{3}$ was used as the solvent. For internal calibration, the solvent signal corresponding to $\mathrm{CDCl}^{3}$ was used. $\delta\left({ }^{1} \mathrm{H}\right)=$ $7.26 \mathrm{ppm}$.

A FTIR spectrometer Bruker Vertex 70 equipped with an attenuated total reflection accessory with thermal control and a diamond crystal (Golden Gate Heated Single Reflection Diamond ATR, Specac-Teknokroma) and equipped with a mid-band liquid nitrogen-cooled mercury-cadmiumtelluride (MCT) detector was used to monitor the evaluation of isocyanate/thiol groups during the isothermal curing at $120^{\circ} \mathrm{C}$. Spectra were collected at this temperature in the absorbance mode at a resolution of $4 \mathrm{~cm}^{-1}$ in the wavelength range of 600 to $4000 \mathrm{~cm}^{-1}$ and 20 scans were averaged for each spectrum. The spectra were corrected for the wavelength dependence of the absorbance in ATR devices. The samples were prepared as detailed in 2.2. Preparation of the formulations. A thin layer of the investigated formulation was put directly on the diamond crystal of the ATR previously heated at $120{ }^{\circ} \mathrm{C}$. 
The conversion $(\alpha)$ of isocyanate groups as function of the time were determined by monitoring the evolution of the peak at $2250 \mathrm{~cm}^{-1}$. The degree of conversion of isocyanate were calculated as:

$$
\alpha=1-\frac{A_{t}}{A_{0}}
$$

where $A$ is the normalized area of the isocyanate band, and the subscripts $t$ and 0 indicate the curing time and at the beginning of the curing, respectively. The peak at $1680 \mathrm{~cm}^{-1}$ (stretch of ester ring of thiol) was used as an internal standard. The band corresponding to the S-H stretch vibration of the thiol group at $2570 \mathrm{~cm}^{-1}$ was only analyzed from a qualitative point of view, due to its weak intensity.

Dynamic mechanical thermal analyses (DMTAs) were performed with a TA Instruments DMA Q800 analyzer. Prismatic rectangular samples (15 x 6 × $\left.1.5 \mathrm{~mm}^{3}\right)$, after isothermal cure, were analyzed by three-point bending at a heating rate of $3^{\circ} \mathrm{C} / \mathrm{min}$ from 35 to $180^{\circ} \mathrm{C}$ using a frequency of $1 \mathrm{~Hz}$ and oscillation of $0.1 \%$ of sample deformation.

The Young's moduli $(E)$ were determined under flexural conditions at $30^{\circ} \mathrm{C}$, with the same clamp and geometry samples, by using a force ramp at a constant rate of $1 \mathrm{~N} / \mathrm{min}$, and ensuring that only the elastic behaviour of the material was evaluated. Three samples of each material were analyzed and the results were averaged. $E$ was calculated from the slope of the linear proportional part of the load-deflection curve according to the following equation:

$$
E=\frac{L^{3} m}{4 b t^{3}}
$$

where $E$ is the elastic modulus of the sample (MPa), $L$ is the support span (mm), $b$ and $t$ are the width and the thickness, respectively, of the sample tested $(\mathrm{mm})$ and $m$ is the gradient of the slope in the linear region $(\mathrm{N} / \mathrm{mm})$.

The thermal stability of the cured samples was studied by thermogravimetric analysis (TGA), using a Mettler TGA/SDTA 851e thermobalance. All experiments were performed under inert atmosphere ( $\mathrm{N}_{2}$ at $\left.100 \mathrm{~mL} / \mathrm{min}\right)$. Pieces of cured samples of 10-15 mg were degraded between 30 and $600^{\circ} \mathrm{C}$ at a heating rate of $10^{\circ} \mathrm{C} / \mathrm{min}$.

Tensile tests at room temperature were carried out by using an electromechanical universal testing machine (Shimadzu AGS-X $10 \mathrm{kN}$ ) at a crosshead speed of $1 \mathrm{~mm} / \mathrm{min}$ and using Type V samples according to ASTM D638-14 standard. Three samples of each material were analyzed and the results were averaged.

Vickers microindentation hardness was measured with a Wilson Wolpert (Micro-Vickers 401 MAV) device following ASTM E384-16 standard procedure. For each material at least 20 
determinations were made with a confidence level of 95\%. The Vickers hardness number (HV) was calculated from the following equation:

$$
H V=\frac{1.854 * F}{d^{2}}
$$

where $F$ is the load applied to the indenter in $\mathrm{kgf}(0.05 \mathrm{kgf})$ and $d$ is the arithmetic mean of the length of the two diagonals of the surface area of the indentation measured after load removal in mm.

\section{Results and Discussion}

\subsection{Calorimetric study of the curing process}

In order to obtain a temporal control of the reaction, we first investigated the curing process in the presence of the acid catalyst mostly used in the preparation of poly(urethane), dibuthyltin dilaurate (DBTDL) and two latent basic catalysts, LC80 and BG1MI, which are an encapsulated imidazole and a tetraphenyl borate imidazolium salt, respectively, which on heating release imidazole [33]. As starting monomers, we selected isophorone diisocyanate (IPDI) and pentaerythritol tetrakis(3-mercaptopropionate) (PETMP) as the thiol. Stoichiometric formulations of comonomers were used in all cases to reach the highest crosslinking density.

Non-isothermal calorimetric studies were performed to analyze the curing evolution of thiolisocyanate formulations with $1 \mathrm{phr}$ of the different catalyst selected. Fig. 1 shows the calorimetric curves for the formulations studied.

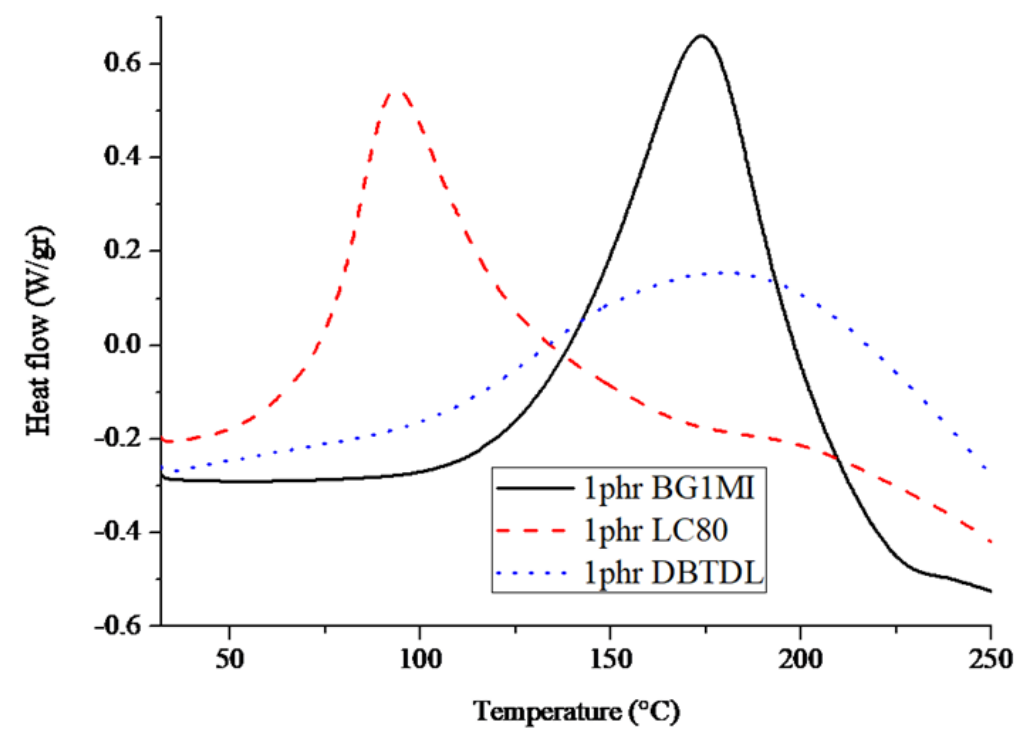

Figure 1. DSC curves of formulations of PETMP-IPDI and 1 phr of the different catalysts selected. 
From this figure, we can observe the different shape of the curves and different initiation temperatures of the curing process for the different catalysts. It is clear that the use of BG1MI allows a more significant control of the initiation, since the curing does not start at room temperature, but at temperatures higher than $100^{\circ} \mathrm{C}$, due to the thermal decomposition of the imidazolium salt that release the base, as represented in Scheme 3 [35]. Once started, the curing is the fastest. Although without enough reliability, we could evaluate in the curves of this figure, an approximate enthalpy of 72 $\mathrm{kJ} / \mathrm{isocyanate}$ equivalent by using BG1MI as the catalyst, $45 \mathrm{~kJ} / \mathrm{i}$.e. with LC80 and $64 \mathrm{~kJ} / \mathrm{i}$.e. when using DBTDL. From these values, it seems to be clear that the curing process starts immediately when using LC89 and DBTDL as catalysts.

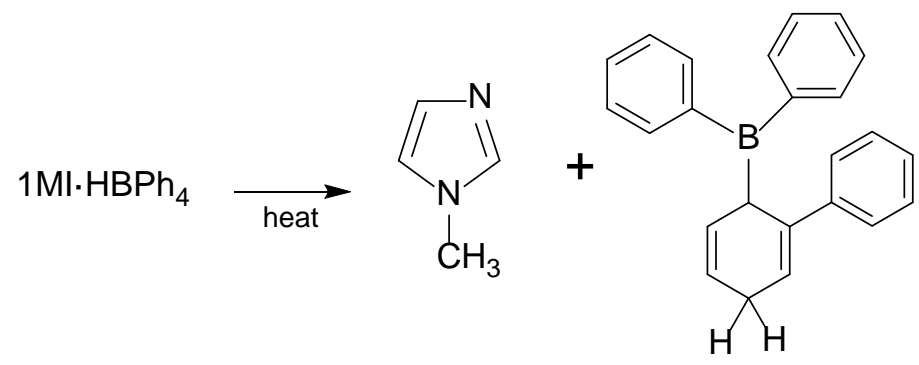

Scheme 3. Thermal activation of the base generator BG1MI

LC80 does not show any latent behavior in this case, since it starts at low temperatures, and even some reaction was appreciated at room temperature by a slight increase in the viscosity on preparing the sample at room temperature. This makes the evaluation of the curing enthalpy unreliable. This behavior is contrary to that observed in thiol-epoxy systems in which the samples could be stored during more than one week at $35^{\circ} \mathrm{C}$ without any appreciable change [32]. The use of DBTDL as the catalyst leads to a broad curve, starting at room temperature without any recovery of the base-line, indicating that the curing is still not finished at $250^{\circ} \mathrm{C}$ or that some degradation processes occur.

The effect of the variation of the proportion of the catalyst was also investigated. The calorimetric curves for the different catalyst are collected in Figure 2. The shape of the curves did not allow us to determine accurately the enthalpy released by gram of mixture or by isocyanate mol. 

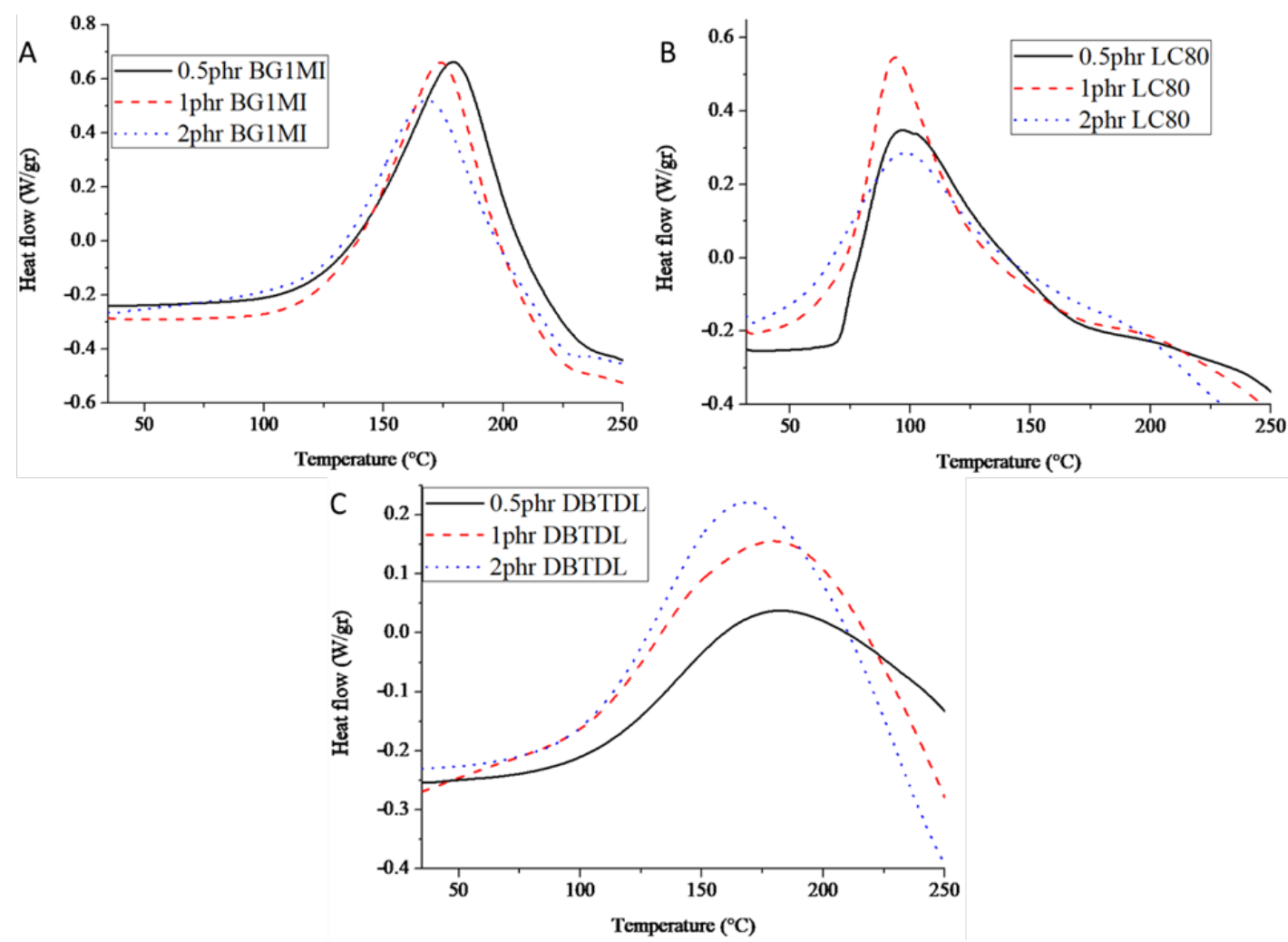

Figure 2. DSC curves of formulations of PETMP-IPDI and the different catalysts in proportions of 0.5, 1 and 2phr.

In Figure 2A we cannot see many differences among the curing exotherms on varying the amount of BG1MI and in all cases the latent character was maintained. It can be only noticed a slight decrease of the temperature of the maximum of the curve on increasing the amount of the catalyst. The decrease of the amount of LC80 to $0.5 \mathrm{phr}$ seems to improve the latency of the system, since the shape of the exotherm changes and it starts at $70^{\circ} \mathrm{C}$. However, the prepared mixture is not stable and begins to react at room temperature after half an hour, probably due to the release of the imidazole by the swelling and partial dissolution of the capsule. The differences observed in Figure 2C on changing the amount of catalyst are mainly related to the curing rate that increases with the DBTDL proportion. Moreover, the curing seems to be finished at $250^{\circ} \mathrm{C}$ when $2 \mathrm{phr}$ of catalyst are used in the formulation, although probably thermal degradation takes place in some proportion since the baseline does not recover.

With the results obtained we investigated the kinetic behaviour of three different aliphatic diisocyanates; IPDI, HMDI and HDI. According to the low stability of LC80 formulations, only DBTDL and BG1MI have been used as the catalyst in a proportion of $0.5 \mathrm{phr}$. It should be commented 
that both LC80 and BG1MI follow the same anionic reaction mechanism, since both release imidazole, but in different conditions. Figure 3 present the calorimetric curing exotherms of these formulations.

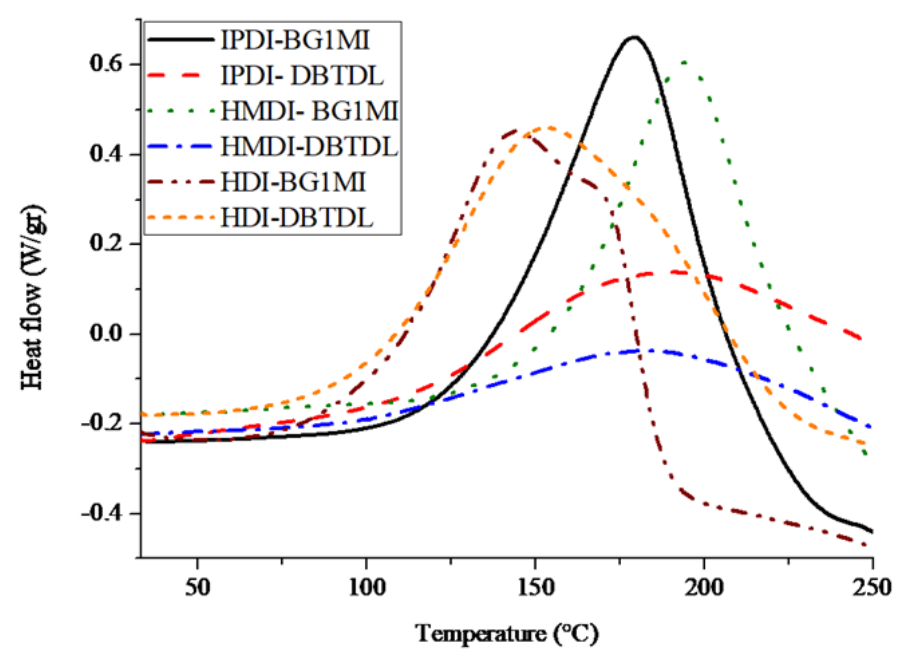

Figure 3. DSC curves of formulations of PETMP-(IPDI/HMDI/HDI) and the catalysts in proportions of $0.5 \mathrm{phr}$.

From this figure, it can be seen that HDI, with a linear structure, is the most reactive monomer with both catalysts and it reacts slowly at temperatures about $75^{\circ} \mathrm{C}$. On the other hand, HMDI reacts at higher temperatures than IPDI in both cases, but this effect is more noticeable in anionic conditions. Whereas HDI cures rapidly with both catalysts, IPDI and HMDI react much faster, once the catalyst is activated, on using the base generator. The use of DBTDL needs higher temperature and longer reaction times to complete the process.

From this study, we can state that from the point of view of the kinetics and easiness in the application of these materials, BG1MI is the most advantageous catalyst and that $0.5 \mathrm{phr}$ seems to be enough to reach a high crosslinking density with all the diisocyanates selected.

\subsection{FTIR study of the curing evolution}

The thiol-isocyanate reaction that takes place in the curing process was followed by FT-IR spectroscopy to investigate not only the kinetics of the reaction but also if the complete formation of poly(thiourethane) networks has been reached.

The FTIR spectra of the IPDI-0.5 phr of BG1MI formulation at different curing times were collected in Figure 4 . In the basis of the calorimetric curve, a temperature of $120^{\circ} \mathrm{C}$ was selected for 
the isothermal curing. The reduction in the isocyanate and thiol peaks, at $2270 \mathrm{~cm}^{-1}$ and $2570 \mathrm{~cm}^{-1}$, respectively, was accompanied by the appearance of $\mathrm{N}-\mathrm{H}$ st. $\left(3350 \mathrm{~cm}^{-1}\right)$ and carbonyl stretching $\left(1670 \mathrm{~cm}^{-1}\right)$ of the thiourethane groups, which are indicative of the network formation. The thiol band is very weak and has no kinetic significance but only qualitative. The carbonyl absorption of the thiourethane group was partially overlapped with the carbonyl ester absorption of the PETMP and the deconvolution has been done in order to extract quantitative information. As we can see in the spectrum registered after $60 \mathrm{~min}$ at $120^{\circ} \mathrm{C}$, some isocyanate absorption still remains, which indicates that this thermal treatment was not enough to reach the complete curing. The fully conversion of isocyanate was achieved after a post-curing treatment at $160^{\circ} \mathrm{C}$ for $1 \mathrm{~h}$. The limited conversion at $120^{\circ} \mathrm{C}$ could be attributed to the vitrification of the material.

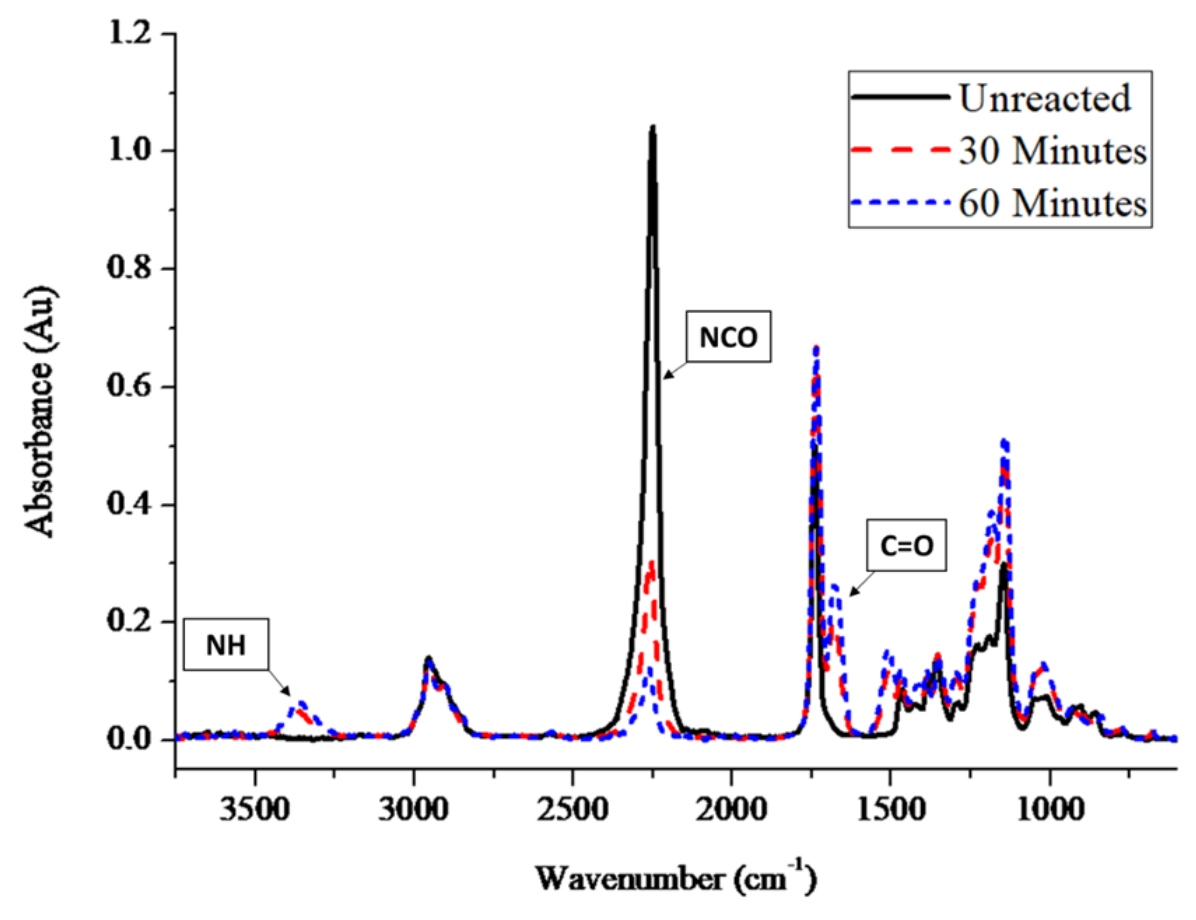

Figure 4. FTIR spectra of the PETMP-IPDI and $0.5 \mathrm{phr}$ of BG1MI at $120^{\circ} \mathrm{C}$.

The evolution of the isocyanate group conversion at the temperature of $120^{\circ} \mathrm{C}$ for the formulation containing a stoichiometric amount of IPDI and PETMP with the different catalyst was determined and represented in Figure 5. 


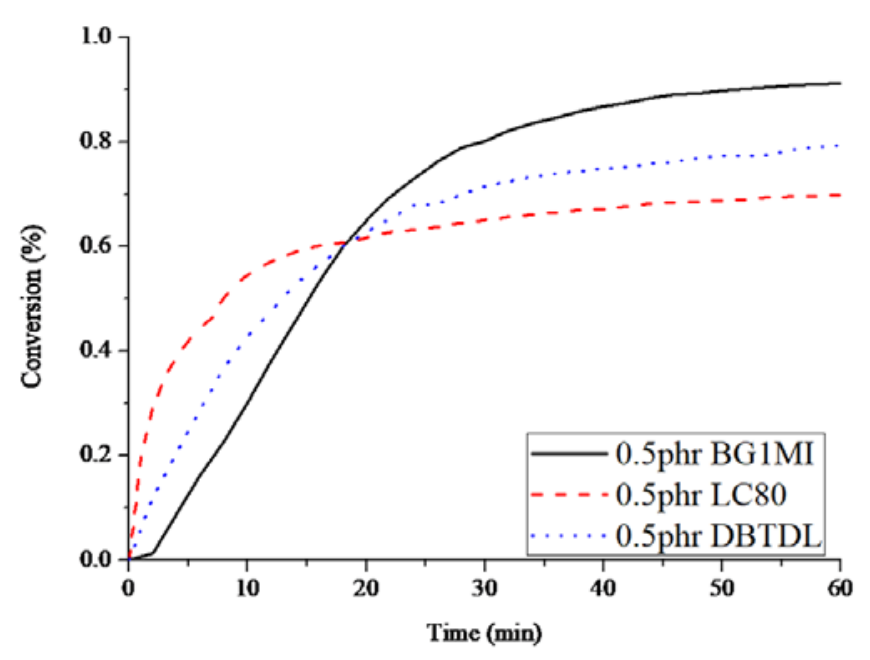

Figure 5. Kinetic profile at $120^{\circ} \mathrm{C}$ of the conversion of NCO group in the formulation of IPDI and PETMP with 0.5 phr of the different catalysts obtained from FTIR analysis

As we can see, with the use of BG1MI as the catalyst a conversion of isocyanate group higher than $90 \%$ was achieved, greater than using the other catalysts. It can also be observed that the reaction with this latent system begins not instantaneously as occurs with formulations catalysed by LC80 or DBTDL, but after a short activation period. The quickest reaction occurs with LC80, but the conversion achieved is quite low. Complete conversion was achieved with all these catalysts when a post-curing at higher temperatures was performed.

From the studies of the curing process by the different techniques, the adequacy of BG1M1 as the catalyst has been confirmed. This catalyst allows a good control of the curing process while reaching the complete curing at moderated temperatures.

\subsection{Thermal characterization of the poly(thiourethane) thermosets}

Although from the point of view of the kinetics and manipulation BG1MI is the most adequate catalyst, the characteristics of the materials prepared with both catalysts can vary notably. Thus, we have investigated the thermomechanical behaviour of all the materials prepared from formulations with the three diisocyanates selected and PETMP with 0.5 phr of DBTDL or BG1MI. Figure 6 shows the $\tan \delta(\mathrm{A})$ and storage modulus (B) curves obtained by DMTA. The main data extracted from these curves are collected in Table 1. 

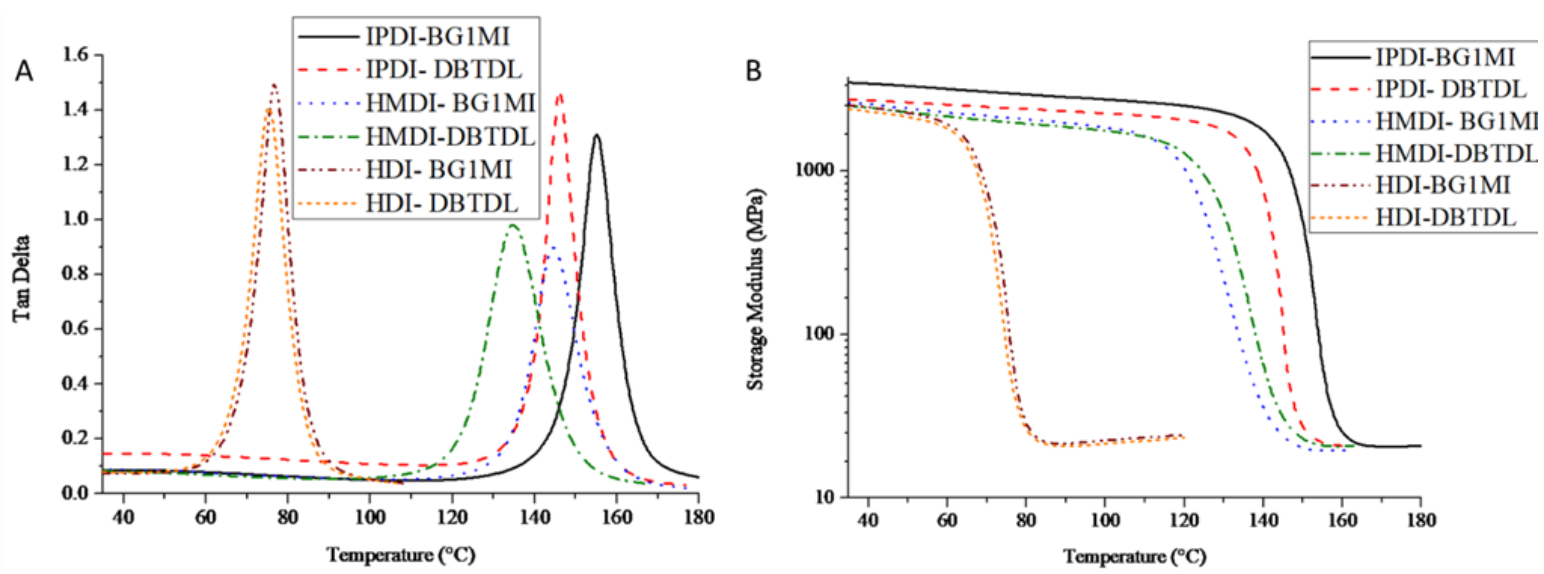

Figure 6. (A) tan $\delta$ and (B) storage modulus against temperature of the different materials prepared with PETMP-IPDI/HMDI/HDI-0.5phr of catalysts

First, it should be mentioned that the curves are very narrow because of the click reaction mechanism. Their good performance without any side-reaction that could lead to unexpected structures is the reason for this homogeneity. The high homogeneity of the material is a crucial aspect for optical applications $[14,15]$. The materials obtained show values of FWHM (width of the curves at half height) in the range between 9 and $16^{\circ} \mathrm{C}$ (see Table 1), which are lower than those obtained by Shin et al. that reach values in the range from 15 to $28{ }^{\circ} \mathrm{C}$ [34]. These authors prepared IPDI/PETMP films by photoinitiation with a base generator but a broader curve with a value of 16 ${ }^{\circ} \mathrm{C}$ was obtained. This result can be justified by the fast curing performed in photochemical conditions.

Table 1. Thermal data obtained from the materials obtained from the different diisocyanates and PETMP with different catalysts at $0.5 \mathrm{phr}$

\begin{tabular}{|c|c|c|c|c|c|c|c|}
\hline Formulation & $\begin{array}{c}T \tan \delta^{\mathrm{a}} \\
\left({ }^{\circ} \mathrm{C}\right)\end{array}$ & $\begin{array}{c}\text { FWHM }^{\mathrm{b}} \\
\left({ }^{\circ} \mathrm{C}\right)\end{array}$ & $\begin{array}{c}\text { Young's } \\
\text { Modulus } \\
\text { (GPa) }\end{array}$ & $\begin{array}{c}E^{, d} \\
(\mathbf{M P a})\end{array}$ & $\begin{array}{l}\mathbf{T}_{2 \%}{ }^{\mathrm{e}} \\
\left({ }^{\circ} \mathrm{C}\right)\end{array}$ & $\underset{\left({ }^{\circ} \mathrm{C}\right)}{T_{\max } f}$ & $\begin{array}{c}\text { Char } \\
\text { Yieldg } \\
\left({ }^{\circ} \mathrm{C}\right) \\
\end{array}$ \\
\hline $\begin{array}{c}\text { IPDI- } \\
0.5 \% B G 1 \mathrm{MI}\end{array}$ & 153 & 11 & 2.8 & 23 & 284 & $\begin{array}{c}305-347- \\
437\end{array}$ & 3.0 \\
\hline $\begin{array}{c}\text { IPDI- } \\
0.5 \% \text { DBTDL } \\
\end{array}$ & 144 & 10 & 2.8 & 21 & 289 & $\begin{array}{c}\text { 308-338- } \\
425\end{array}$ & 4.9 \\
\hline $\begin{array}{c}\text { HMDI- } \\
0.5 \% B G 1 \mathrm{MI}\end{array}$ & 140 & 16 & 2.2 & 19 & 287 & $\begin{array}{c}316-348- \\
453\end{array}$ & 2.9 \\
\hline $\begin{array}{c}\text { HMDI- } \\
0.5 \% \text { DBTDL }\end{array}$ & 135 & 14 & 2.0 & 19 & 289 & $\begin{array}{c}312-337- \\
438\end{array}$ & 4.3 \\
\hline $\begin{array}{c}\text { HDI- } \\
0.5 \% \text { BG1MI }\end{array}$ & 77 & 9 & 2.0 & 24 & 276 & $\begin{array}{c}\text { 304-348- } \\
469 \\
\end{array}$ & 2.2 \\
\hline $\begin{array}{c}\text { HDI- } \\
0.5 \% \text { DBTDL }\end{array}$ & 75 & 10 & 2.0 & 23 & 285 & $\begin{array}{c}317-350- \\
467\end{array}$ & 2.4 \\
\hline
\end{tabular}

a Temperature of maximum of the tan $\delta$ at $1 \mathrm{~Hz}$.

${ }^{\mathrm{b}}$ FWHM stands by full width at half maximum.

c Young's modulus determined at $30^{\circ} \mathrm{C}$ under flexural conditions

${ }^{\mathrm{d}}$ Relaxed modulus determined at the $\mathrm{T}_{\tan \delta}+40^{\circ} \mathrm{C}$ (in the rubber state). 
e Temperature of $2 \%$ of weight loss

${ }^{\mathrm{f}}$ Temperatures of the maximum rate of the three steps of degradation

g Char residue at $600^{\circ} \mathrm{C}$

The values of the temperature of the maximum of $\tan \delta$ change not only with the monomer structure but also in some materials depend on the catalyst used. The flexible structure of HDI leads to lower $\tan \delta$ temperatures in comparison to the materials containing rigid cycloaliphatic groups, without much difference on changing the catalyst. However, IPDI and HMDI derived materials show variations on changing the catalyst, leading to higher temperature of $\tan \delta$ values when the base generator was used. Although a post-curing at $170{ }^{\circ} \mathrm{C}$ for 3 hours or an increase in the DBTDL proportion were tested, the crosslinking density seems to be limited and this value did not increase. This fact can be related to the kinetic evolution of the curing process observed by DSC (Fig. 3), in which DBTDL cures at higher temperature and the enthalpy released seems to be lower. IPDI derived materials present the highest $\mathrm{T}_{\tan \delta}$ due to their higher compact and rigid network.

In Figure 6B the evolution of storage modulus with the temperature is represented for all the samples. As we can see, the relaxation of the material takes place sharply with a significant change in the moduli in glass and rubber state. There are no big differences in the relaxed moduli (see Table 1), but a certain correlation with the molecular weight between crosslinks can be appreciated. The highest molecular weight between crosslinks of HMDI materials leads to the lowest modulus. Although the difference is only slight, the use of DBTDL leads to a lower value, according to the lower crosslinking density achieved with this catalyst.

The Young's modulus determined at $30^{\circ} \mathrm{C}$ in bending mode yields the highest value for the most rigid network structure obtained from IPDI formulations.

Some authors studied the degradation of poly(urethane)s [20] and poly(thiourethane-urethane)s [36] thermoplastics and a complex degradation mechanism, with two or three degradation steps, was reported. Depending on the aliphatic or aromatic character of the urethane structure the reversion of urethane to isocyanate and hydroxyl groups takes place between 120 to $250^{\circ} \mathrm{C}$, being the aliphatic ones the most stable. The first degradation step was attributed to this degradative process. The second step, at higher temperature, corresponds to a typical $\beta$-elimination process, if there are hydrogen atoms in $\gamma$-position. This process leads to the formation of a carbamic acid and an olefin. Carbamic acid, which is unstable, release carbon dioxide and the corresponding amine is formed. It has been reported that this process can lead to one or two degradation steps [37]. 
In the poly(thiourethane) thermosets prepared in the present work, the DTG curves (derivative of TGA degradation curves) clearly present three different degradation steps, with no much differences on changing the structure of the isocyanate and the catalyst used in their curing. Figure 7 presents the DTG curves of the materials prepared and the most representative degradation data are collected in Table 1.
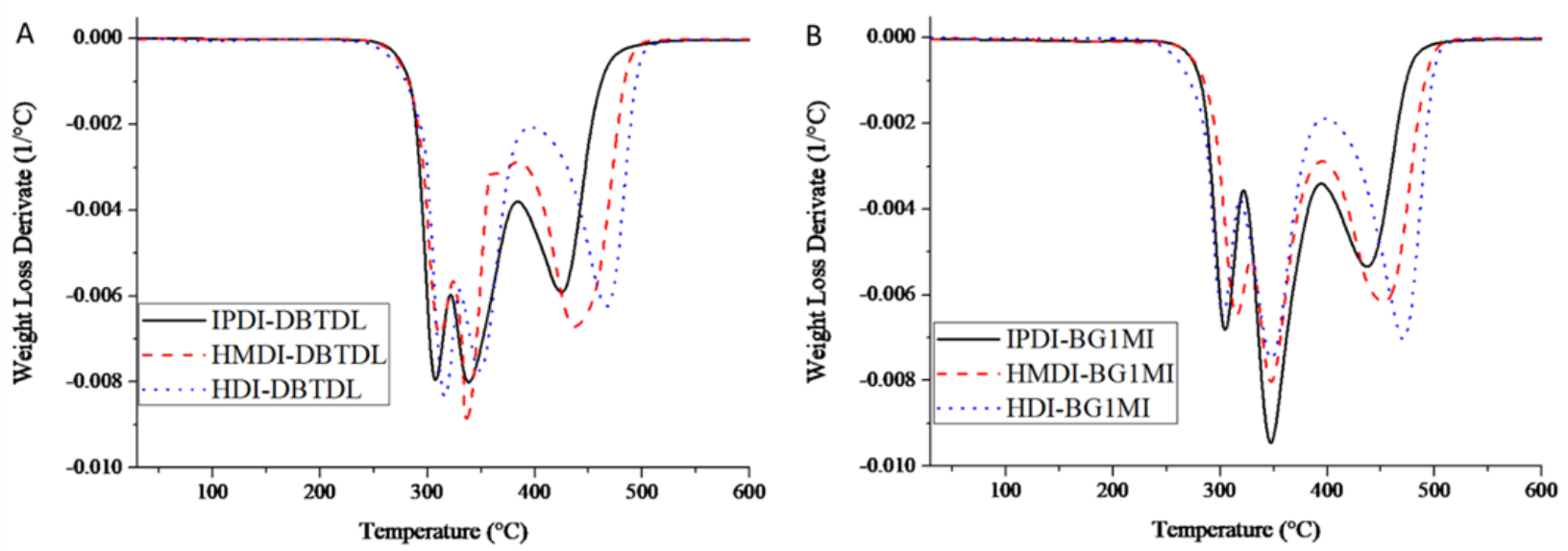

Figure 7. DTG curves of PETMP-(IPDI/HDMI/HDI) thermosets using $0.5 \mathrm{phr}$ of BG1MI (A) and $0.5 \mathrm{phr}$ of DBTDL as the catalysts.

As we can see in the figure and table, the first degradation step begins at about $300^{\circ} \mathrm{C}$, which indicates that poly(thiourethanes) are more stable than their corresponding oxygen analogues, which decompose at about $250^{\circ} \mathrm{C}$ [20]. In our case, two possible $\beta$-elimination processes can occur, because not only thiourethanes are present but also ester groups from the tetrathiol structure. Both $\beta$ elimination processes could overlap and contribute to the degradation maximum at about $350^{\circ} \mathrm{C}$. The degradation peak at the highest temperature must correspond to the fully degradation of the network. If we look to these temperatures of the maximum rate of degradation (Table 1), we can see that the values for the last step are more dependent on the urethane structure. In general, char yields are slightly higher for those materials prepared with DBTDL and this catalyst seems to reduce the temperature of the urethane decomposition through $\beta$-elimination.

From these studies, we can state that these materials exhibit a quite good thermal stability, higher than the poly(urethane) analogues, with decomposition temperatures higher than $280{ }^{\circ} \mathrm{C}$, with the exception of the material obtained from HDI and the base generator that loss a $2 \%$ of weight at $276^{\circ} \mathrm{C}$, attributable to its linear aliphatic structure. 


\subsection{Mechanical Characterization of the Materials}

Microindentation hardness tests and tensile tests at break have been performed for all the thermosets prepared. The data extracted from these tests are listed in Table 2.

Table 2. Mechanical data determined from the materials obtained with the different diisocyanates and different catalysts

\begin{tabular}{|c|c|c|c|c|}
\hline Formulation & $\mathbf{V H}^{\mathbf{a}}$ & $\begin{array}{c}\text { Tensile } \\
\mathbf{M o d u l u s} \\
\mathbf{( G P a )}\end{array}$ & $\begin{array}{c}\mathbf{\sigma b}^{\mathbf{c}} \\
\mathbf{( M P a )}\end{array}$ & $\begin{array}{c}\boldsymbol{\varepsilon}_{\mathbf{b}}^{\mathbf{d}} \\
\mathbf{( \% )}\end{array}$ \\
\hline IPDI-0.5\%BG1MI & $19.6 \pm 0.7$ & $2.1 \pm 0.1$ & $72.9 \pm 2.6$ & $5.8 \pm 0.4$ \\
\hline IPDI-0.5\%DBTDL & $19.2 \pm 0.5$ & $2.1 \pm 0.1$ & $76.1 \pm 2.2$ & $6.3 \pm 0.8$ \\
\hline HMDI-0.5\%BG1MI & $16.1 \pm 0.7$ & $1.7 \pm 0.1$ & $67.1 \pm 1.7$ & $6.4 \pm 0.4$ \\
\hline HMDI-0.5\%DBTDL & $15.9 \pm 0.6$ & $1.7 \pm 0.1$ & $64.1 \pm 2.5$ & $6.6 \pm 0.8$ \\
\hline HDI-0.5\%BG1MI & $13.2 \pm 0.8$ & $1.7 \pm 0.1$ & $47.3 \pm 1.6$ & $3.8 \pm 0.2$ \\
\hline HDI-0.5\%DBTDL & $12.4 \pm 0.6$ & $1.7 \pm 0.1$ & $44.6 \pm 1.4$ & $3.2 \pm 0.1$ \\
\hline
\end{tabular}

${ }^{a}$ Vickers microindentation hardness.

${ }^{\mathrm{b}}$ Tensile modulus at room temperature.

${ }^{\mathrm{c}}$ Stress at break.

${ }^{\mathrm{d}}$ Deformation at break.

Mechanical characteristics are fully dependent on the chemical structure of the network and crosslinking densities. The higher values of microindentation hardness were obtained for IPDI derived thermosets, because of their rigid structure. On increasing the flexibility of the selected isocyanate a decrease in microindentation hardness was observed and the lowest values were obtained for HDI derived thermosets, with a long aliphatic chain between thiourethane moieties.

With respect to the stress-strain behaviour, all these materials are relatively rigid and does not experiment appreciable plastic deformations at room temperature due to their crosslinked character. Although a similar influence on changing the monomer structure was expected in the tensile modulus, we have not noticed any difference between materials prepared from HMDI and HDI due to the glassy state at room temperature. However, the rigid and compact structure of IPDI leads to a notable higher modulus for this material. These results are in accordance with those obtained from DMTA tests. The most relevant differences between these materials can be observed in the stress and strain at break values. The tensile strength is the highest when the rigid IPDI moiety was in the network structure and the lowest with the linear and aliphatic HDI. With respect to the strain at break, related to the ductility, the poly(thiourethanes) based on HMDI present the maximum value, since materials based on HDI suffer a more easy breakage at lower stress due to their significantly lower strength. The type of catalyst used seems not to influence in a significant manner the tensile characteristics of the thermosets as the values presented are similar. 


\section{Conclusions}

The use of 1-methylimidazolium tetraphenylborate (BG1MI), as a latent base catalyst, allowed a much better control of the reaction of aliphatic diisocyanates and tetrathiol monomer to prepare poly(thiourethane) networks than the commonly used dibutyltin dilaurate (DBTDL). The reaction begins at $120^{\circ} \mathrm{C}$ and occurs rapidly. LC80, which is an encapsulated imidazole, did not show the expected latent behaviour in this curing process.

The conversion of isocyanates in the reactive formulations reached after 1 hour at $120^{\circ} \mathrm{C}$ with BG1MI as the catalyst was higher than 90\%, whereas the use of DBTDL reaches only a conversion of $75 \%$. On increasing the temperature, the fully conversion was achieved with both catalysts.

Different poly(thiourethane) thermosets were prepared from three different isocyanates: isophorone diisocyanate (IPDI), 4,4'-methylene bis(cyclohexy isocyanate) (HMDI) and hexamethylene diisocyanate (HDI) and a tetrathiol, pentaerythritol tetrakis(3-mercaptopropionate) (PETMP) with DBTDL and BG1MI, as acid and basic catalysts, respectively.

All materials prepared showed very narrow $\tan \delta$ curves, which stands for highly homogeneous networks. $T_{g}$ values increase with increasing the rigidity of the isocyanate monomer, reaching a maximum value of $150^{\circ} \mathrm{C}$ for IPDI derived materials. The use of the base generator as the catalyst leads to materials with higher $\mathrm{T}_{\mathrm{g}}$.

The initial degradation of these materials occurred at higher temperatures than the related poly(urethanes). Their degradation curves allowed to detect three different degradation mechanisms. The first one, at the lowest temperature, corresponds to the reversion of thiourethanes to form isocyanate and thiol groups. On increasing the temperature, the $\beta$-elimination of the remaining thiourethanes and ester groups of the thiol structure occurs and then, at the highest temperature, the complete degradation of the network structure could be observed.

The mechanical behaviour of the prepared materials could be correlated to the network structure, according to the rigid or flexible character of the starting diisocyanate monomer. IPDI derived materials are more strength, rigid and harder than those derived from HDI, which present the worst mechanical performance. The materials obtained from the latent catalyst have similar mechanical characteristics to the ones obtained by using the commonly used DBTDL. 


\section{Acknowledgments}

The authors would like to thank MINECO (Ministerio de Economia, Industria y Competitividad, MAT2017-82849-C2-1-R and 2-R) and Generalitat de Catalunya (2017-SGR-77) for their financial support.

\section{Declarations of interest}

None.

\section{Data availability}

The raw/processed data required to reproduce these findings cannot be shared at this time as the data also forms part of an ongoing study.

[1] H.C. Kolb, M.G. Finn, K.B. Sharpless, Click chemistry: diverse chemical function from a few good reactions, Angew. Chem. 40 (2001) 2004-2021.

[2] X. Ramis, X. Fernández-Francos, S. De La Flor, F. Ferrando, A. Serra, Click-Based Dual-Curing Thermosets, in Q. Guo, (Ed.), Thermosets: Structure, Properties and Applications, 2nd ed., Elsevier, Amsterdam, 2018, Chap. 16.

[3] C.E. Hoyle, C.N. Bowman, Thiol-Ene click chemistry, Angew. Chem. 49 (2010) 1540-1573.

[4] S. Chandrasekaran, Click Reactions in Organic Synthesis, Wiley-VCH, Weinheim, Germany, 2016.

[5] A.B Lowe, Thiol-ene “click” reactions and recent applications in polymer and materials synthesis, Polym. Chem. 1 (2009) 17-36.

[6] H. Li, B. Yu, H. Matushima, C.E. Hoyle, A.B. Lowe, The thiol-isocyanate click reaction: facile and quantitative access to $\omega$-end-functional poly(N,N-diethylacrylamide) synthesized by RAFT radical polymerization, Macromolecules 42 (2009) 6537-6542.

[7] E. Dyer, D.W. Osborne, The synthesis of polythiolcarbamates, J. Polym. Sci. 47 (1960) 361-371.

[8] H.-W. Engels, H.-G. Pirkl, R. Albers, R. W. Albach, J. Krause, A. Hoffmann, H. Casselmann, J. Dormish, Polyurethanes: versatile materials and sustainable problem solvers for today's challenges, Angew. Chem. 52 (2013) 9422-9441.

[9] A.B. Lowe, C.N. Bowman, Thiol-X Chemistries in Polymer and Materials Science, RSC Polymer Chemistry Series 6, Croydon, UK, 2013. 
[10] K. Strzelec, N. Baczek, S. Ostrowska, K. Wasikowska, M.I. Szynkowska, J. Grams, Synthesis and characterization of novel polythiourethane hardeners for epoxy resins, C. R. Chimie 15 (2012) 1065-1071.

[11 ] Q. Li, H. Zhou, A. Wicks, C.E. Hoyle, D.H. Magers, H.R. McAlexander, Comparison of small molecule and polymeric urethanes, thiourethanes, and dithiourethanes: hydrogen bonding and thermal, physical, and mechanical properties, Macromolecules 42 (2009) 1824-1833.

[12] C. Lu, Z. Cui, Z. Li, B. Yang, J. Shen, High refractive index thin films of ZnS/polythiourethane nanocomposites, J. Mater. Chem. 13 (2003) 526-530.

[13] C. Lu, C. Guan, Y. Liu, Y. Cheng, B. Yang, PbS/Polymer nanocomposite optical materials with high refractive index, Chem. Mater. 17 (2005) 2448-2454.

[14] B. Jaffrennou, N. Droger, F. Mechin, J.-L. Halary, J.-P Pascault, Characterization, structural transitions and properties of a tightly crosslinked polythiourethane network for optical applications, e-Polym. 82 (2005) 1618-7229.

[15] N.G. Ireni, R. Narayan, P. Basak, K.V.S.N. Raju, Poly(thiourethane-urethane-urea) as anticorrosion coatings with impressive optical properties, Polymer 97 (2016) 370-379.

[16] J. Tan, C. Li, H. Li, H. Zhang, J. Gu, B. Zhang, H. Zhang, Q. Zhang, Water-borne thiolisocyanate click chemistry in microfluidics: rapid and energy-efficient preparation of uniform particles, Polym. Chem. 6 (2015) 4366-47373.

[17] C. Li, J. Tan, H. Li, D. Yin, J. Gu, B. Zhang, Q. Zhang, Thiol-isocyanate click reaction in a Pickering emulsion: a rapid and efficient route to encapsulation of healing agents, Polym. Chem. 2015, 6, 7100-7111.

[18] J. Yan, S. Ariyasivam, D. Weerasinghe, J. He, B. Chisholm, Z. Chen, D. Webster, Thiourethane thermoset coatings from bio-based thiols. Polym. Int. 61 (2012) 602-608.

[19] X. K. D. Hillewaere, R. F. A. Teixeira, L-T. T. Nguyen, J. A. Ramos, H. Rahier, F. E. Du Prez, Autonomous Self-Healing of Epoxy Thermosets with Thiol-Isocyanate Chemistry, Adv. Funct. Mater. 24 (2014) 5575-5583.

[20] E. Delebecq, J.P. Pascault, B. Boutevin, F. Ganachaud, On the versatility of urethane/urea bonds: reversibility, blocked isocyanate, and non-isocyanate polyurethanes, Chem. Rev. 113 (2012) 80-118. [21] A.L. Silvia, J.C. Bordado, Recent developments in polyurethane catalysis: catalytic mechanism review. Cat. Rev. - Sci. Eng. 46 (2004) 31-51.

[22] H. Sardon, A. Pascual, D. Mecerreyes, D. Taton, H. Cramail, J.L. Hendirck, Synthesis of polyurethanes using organocatalysis: a perspective, Macromolecules 48 (2015) 315-3165. 
[23] S. Kuypers, S.K. Paramanik, L. D’Olieslaeger, G. Reekmans, M. Peters, J. D'Haen, D. Vanderzande, T. Junkers, P. Adriaensens, A. Ethirajan, Interfacial thiol-isocyanate reactions for functional nanocarriers: a facile route towards tunable morphologies and hydrophilic payload encapsulation, Chem. Commun. 51 (2015) 15858-15861.

[24] H. Salmi, X. Allonas, C. Ley, Polythiourethane networks catalyzed by photobase generators, Prog. Org. Coatings 100 (2016) 81-85.

[25] J. Shin, H. Matsushima, M.C. Comer, C.N. Bowman, C.E. Hoyle, Thiol-Isocyanate-Ene ternary networks by sequential and simultaneous thiol click reactions, Chem. Mater. 22 (2010) 2616-2625. [26] J. Shin, H. Matsushima, J.W. Chan, C.E. Hoyle, Segmented polythiourethane elastomers through sequential thiol-ene and thiol-isocyanate reactions. Macromolecules, 42 (2009) 3294-3301.

[27] S. Chatani, R. Sheridan, M. Podgorski, D.P. Nair, C.N. Bowman, Temporal control of thiol-click chemistry. Chem. Mater. 25 (2013) 3897-3901.

[28] H. Matsushima, J. Shin, C.N. Bowman, C.E. Hoyle, Thiol-isocyanate-acrylate ternary networks by selective thiol-click chemistry, J. Polym. Sci. Part A. Polym. Chem. 48 (2010) 3255-3264.

[29] A. Kultys, M. Rogulska, S. Pikus, The synthesis and characterization of new thermoplastic poly(thiourethane-urethane)s, J. Polym. Sci. Part A. Polym. Chem. 46 (2008) 1770-1782.

[30] A.Basterretxea, Y. Haga, A. Sanchez-Sanchez, M. Isik, L. Irusta, M. Tanaka, K. Fukushima, H. Sardon, Biocompatibility and hemocompatibility evaluation of polyether urethanes synthesized using DBU organocatalyst. Eur. Polym. J. 84 (2016) 750-758.

[31] Official Journal of the European Union 1.4.2010. Commission Regulation No 276/2010

[32] D. Guzmán, X. Ramis, X. Fernández-Francos, A. Serra, New catalysts for diglycidyl ether of bisphenol A curing based on thiol-epoxy click reaction, Eur. Polym. J. 59 (2014) 377-386.

[33] O. Konuray, N. Areny, J.M. Morancho, X. Fernández-Francos, A. Serra, X. Ramis, Preparation and characterization of dual-curable off-stoichiometric amine-epoxy thermosets with latent reactivity. Polymer 146 (2018) 42-52.

[34] J. Shin, J. Lee, H.M. Jeong, Properties of polythiouretanes prepared by thiol-isocyanate click reaction, J. Appl. Polym. Sci. 135 (2018) 1-8.

[35] X. Sun, J.P. Gao, Z.Y. Wang, Bicyclic guanidinium tetraphenylborate: a photobase generator and a photocatalyst for living anionic ring-opening polymerization and cross-linking of polymeric materials containing ester and hydroxy groups, J. Am. Chem. Soc. 130 (2008) 8130-8131.

[36] M. Rogulska, A. Kultys, E. Olszewska, New thermoplastics poly(thiourethane-urethane) elastomers based on hexane-1,6-diyl diisocyanate (HDI), J. Therm. Anal. Calorim. 114 (2013) 903916. 
[37] G. Trovati, E.A. Sanches, S.C. Neto, Y.P. Mascarenhas, G.O. Chierice, Characterization of polyurethane resins by FTIR, TGA, and XRD, J. Appl. Polym. Sci. 115 (2010) 263-268. 